

\title{
Deformation forces in promolecules revisited: Binding of homonuclear diatomic molecules and calculation of stretching vibrational frequencies in diatomic and larger systems
}

Patrick Chaquin, Peter Reinhardt

\section{- To cite this version:}

Patrick Chaquin, Peter Reinhardt. Deformation forces in promolecules revisited: Binding of homonuclear diatomic molecules and calculation of stretching vibrational frequencies in diatomic and larger systems. Computational and Theoretical Chemistry, 2016, 1096, pp.33 - 39. 10.1016/j.comptc.2016.09.031 . hal-01383197

\section{HAL Id: hal-01383197 \\ https://hal.sorbonne-universite.fr/hal-01383197}

Submitted on 18 Oct 2016

HAL is a multi-disciplinary open access archive for the deposit and dissemination of scientific research documents, whether they are published or not. The documents may come from teaching and research institutions in France or abroad, or from public or private research centers.
L'archive ouverte pluridisciplinaire HAL, est destinée au dépôt et à la diffusion de documents scientifiques de niveau recherche, publiés ou non, émanant des établissements d'enseignement et de recherche français ou étrangers, des laboratoires publics ou privés. 


\title{
Deformation forces in promolecules revisited : binding of homonuclear diatomic molecules and calculation of stretching vibrational frequencies in diatomic and larger systems.
}

Patrick Chaquin $^{a, b}$ and Peter Reinhardt ${ }^{a, b}$

[a] Sorbonne Universités, UPMC Univ Paris 06, UMR 7616, Laboratoire de Chimie Théorique, F-75005 Paris, France.

[b] CNRS, UMR 7616, Laboratoire de Chimie Théorique, F-75005 Paris, France

Mail: Patrick.Chaquin@upmc.fr, Peter.Reinhardt@upmc.fr

FAX: +331442741 17

\begin{abstract}
Internuclear forces in a molecule, as an integral over a geometrical factor and the electron density, can be decomposed into a part having as origin promolecular densities of the participating atoms, and a contribution from the deformation density. At the hand of homo-nuclear diatomic molecules we show that the binding energy is linked to the deformation force except for transition metal dimers. However, vibrational frequencies involving pure bond-length variations are rather well reproduced, even for heteroatomic diatomics. For larger assemblies, frequencies for bending modes are underestimated, but the model may serve for a rough analysis of a vibrational spectrum.
\end{abstract}

\section{Introduction}

Following the distinction introduced by Berlin [1], the formation of a chemical bond can be considered from two different points of view. The bonding refers to the variation of energy 
when two atoms or fragments come from infinite separation to their equilibrium distance $R_{e}$. The binding refers to the force exerted on nuclei which should be zero at $\mathrm{R}_{\mathrm{e}}$. In this situation, the attractive force exerted by the electron density on the nuclei exactly cancels their mutual repulsion. The study of chemical binding in terms of forces was the subject of active research from around the 1960 to -1980 [2] and then for a time almost abandoned. A recent renewal is this area can be nevertheless observed, for the study as well as the chemical bonds $[3,4,5]$ as various chemical properties [6] and chemical reactivity [7].

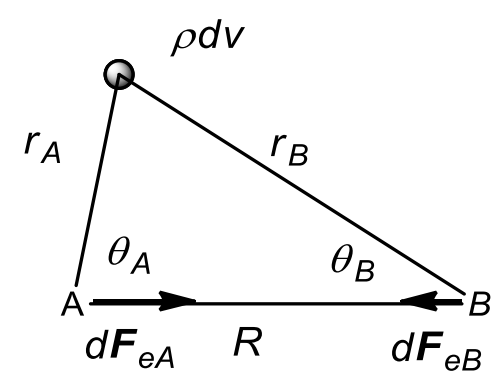

Scheme 1

Using the Hellmann-Feynman theorem, which describes, for a wavefunction satisfying Schrödinger's equation, the force on each nucleus separately in terms of classical electrostatics, Berlin deduced that the force on a chemical bond between two atoms may be decomposed into equal parts arising from each atom. The force $F_{R}$ attracting or repelling the two nuclei is (using the notations of scheme 1):

$$
F_{R}=\frac{Z_{A} Z_{B}}{R^{2}}-\int\left[\frac{1}{2} \frac{Z_{A}}{r_{A}^{2}} \cos \theta_{A}+\frac{1}{2} \frac{Z_{B}}{r_{B}^{2}} \cos \theta_{B}\right] \rho(x, y, z) d v=\frac{Z_{A} Z_{B}}{R^{2}}-\frac{1}{2} \int f(x, y, z) \rho(x, y, z) d v
$$

as (i) a nuclear term and (ii) an integral over the total density multiplying a purely geometrical factor, independently whether atom A or atom B is displaced for varying the interatomic distance $R$. We use the negative sign to indicate that the electron density is positive, however the electron charge is negative. In the formula the two nuclei contribute with equal weights $1 / 2$. This is not necessary, as has been criticized by Silberbach [8], pointing out that any arbitrary partition $Z_{A} \rightarrow \alpha Z_{A}$ and $Z_{B} \rightarrow \beta Z_{B}$ with $\alpha+\beta=1$ leads to the same total force, despite different geometrical functions $f(x, y, z)$. As a matter of fact, the HellmannFeynman theorem uses the variation of the total energy with respect to the displacement of each atom separately, whereas the Berlin expression considers the force with respect to the 
variation of the interatomic distance. Hinze [9] argued from the invariance of physical properties when permuting $A$ and $B$ that the choice of Berlin may be justified which allows us to adopt this expression in the present study.

The generalization of Berlin's formula towards molecules with more than two atoms may be obtained, as advocated by Wang and Peng [10], by looking at the variation of the total energy upon a global scaling of all nuclear coordinates with respect to the geometrical centre (arithmetic average of all nuclear positions, without any weighting by mass of charges of the nuclei).

Having thus the purely geometrical factor of Berlin in mind, we may look at a possible decomposition of the total density when forming a chemical bond. The total density $\rho$ can be written as $\rho_{0}+\Delta \rho$ where $\rho_{0}$ is an arbitrary reference density. If we take as $\rho_{0}$ the promolecular density, $\Delta \rho$ is known as the "deformation density". Following the pioneering work of Daudel [11] and Bader et al. [12, 13, 14], this notion has been subject of a number of studies and discussions. In particular, it was pointed out that the deformation density depends on the choice of the promolecular density [15]: a spherical density, or a density of atoms "oriented" according to the bonding direction, in addition possibly hybridized [16]. It is worth recalling that the promolecular density comes from atomic contributions, calculated separately and once for each type of atom, whereas the deformation density cannot be decomposed into atomic contributions, but has to be evaluated from the overall molecular density. Beyond the simple shape or the contribution to the total energy (like in densityfunctional theory as implemented in the ADF program of Baerends et al. [17]), in comparison, less attention has been devoted to the change in the electronic force resulting from this deformation $\Delta \rho$. Bader showed [13] that it is correlated to the dissociation energy in a series of first row symmetrical diatomic molecules and ions. Hirshfeld [18] developed a similar idea by computing a "migration field" $E_{\Delta}$, difference between a promolecular electric field exerted on a nucleus, and the actual value of this field in the system (which is zero at $R_{e}$ ). From a sample of first row $A_{2}$ and $A H$ molecules, a linear correlation was also found between $\mathrm{E}_{\Delta}$ and the bond dissociation energy.

In the present paper we will, on one hand, extend the calculation of the force associated to the density deformation to a large panel of 30 diatomic homonuclear molecules including 
transition metal dimers in order to check their correlation with dissociation energy. In addition, we calculated in a few cases the force variation resulting from electron deformation of diatomic, "proionic" molecules, i.e. the density of ion pairs A+ A-, with the intention to see whether for instance a $F-F$ charge-shift bond $[19,20]$ may be anticipated from a simple, promolecular picture, despite the favourable dissociation channel into neutral atoms.

On the other hand we will look for vibrational frequencies of diatomic and larger molecules or atomic assemblies, using only promolecular atomic densities.

\section{Calculation details}

Assuming spherical promolecular densities has the advantage that only the charge contained in a sphere of radius $R_{e}$ contributes to the promolecular interaction. To obtain thus spherically symmetric atomic densities, Hartree-Fock calculations are carried out with a locally modified version of the atomic program of Roos and Clementi [21], with aug-cc-pvtz basis sets when available or $6-311+G^{* *}$ in the other cases. We may integrate the orbitals and the corresponding density over a limited sphere through analytic formulæ, for Gaussian or Slater-type functions, or by numerical integration, and obtain thus the effective charge $Q$ within a sphere of given radius. We observed, by the way, that the numerical integration yields more stable results for densities far from the nucleus, as the analytical formulae imply large terms cancelling each other. Correlation effects, important for the total energy, seem less crucial here. For bond lengths, experimental values have been used where available, or DFT-optimized structures elsewhere. Dissociation energies for diatomic molecules are taken from the literature $[22,23,24,25]$.

Vibration frequencies in polyatomic systems have been computed from force constants by diagonalization of the hessian matrix using generalized (mass-weighted) coordinates. The corresponding code has been developed using the implicit-reference-to-parameters tool IRPF90 [26], and uses the tabulated integrated atomic densities with a spline interpolation. All relevant data can be obtained from the authors upon request. 


\section{Results and discussion}

\subsection{Deformation forces of homonuclear diatomic molecules}

The deformation force is defined here as the difference between the force exerted by the exact electron density $\rho$ on nuclei and a "promolecular force", taken as the force exerted by the spherically averaged atomic densities $\rho_{0}$ at $R_{e}$. The total density is $\rho=\rho_{0}+\Delta \rho$.

From the Berlin relation we write at the equilibrium geometry:

$$
\begin{gathered}
0=F_{R A}=\frac{Z_{A} Z_{B}}{R_{e}^{2}}-\frac{1}{2} \int f(x, y, z) \rho d v=\frac{Z_{A} Z_{B}}{R_{e}^{2}}-\frac{1}{2} \int f(x, y, z)\left(\rho_{0}+\Delta \rho\right) d v \\
=\frac{Z_{A} Z_{B}}{R_{e}^{2}}-\frac{1}{2} \int f(x, y, z) \rho_{0} d v+\Delta F
\end{gathered}
$$

thus three contributions as nuclear, promolecular and deformation part, which sum up to zero.

$$
\frac{Z_{A} Z_{B}}{R_{e}^{2}}-F_{0}+\Delta F=0
$$

The force exerted by the spherical electronic density of atom A on its own nucleus is zero. For a homonuclear diatomic molecule $F_{0}$ is thus equal to the classical electrostatic force exerted by the spherical electron density of atom B on nucleus $A$, and vice versa, compensating the factor $1 / 2$. For a spherical density, only the charge $Q_{B}$ within a sphere up to $R_{e}$, contributes to the force, and may be calculated from the Gauss-Ostrogradski theorem:

$$
\begin{gathered}
Q_{B}=-\int_{0}^{R_{e}} \rho_{0 B} d v \\
F_{0}=\frac{-Z_{A}}{R_{e}^{2}} \int_{0}^{R_{e}} \rho_{0 B} d v
\end{gathered}
$$

as if the charge $Q_{B}$ within the sphere were concentrated in point $B$. Note that $Q_{B}$ is less than the total electronic charge $Z_{B}$ of atom $B$, and thus $F_{0}<Z_{A} Z_{B} / R_{e}{ }^{2}$ : the total promolecular force is always repulsive. As a consequence, at equilibrium distance, the deformation force

$$
\Delta F=\frac{-Z_{A}}{R_{e}^{2}}\left(Z_{B}-Q_{B}\right)=\frac{-Z^{2}}{R_{e}^{2}}\left(1-\frac{Q}{Z}\right) \text { Eq. } 1
$$


is a negative quantity to compensate the promolecular repulsion. We dropped the indices $A$ and B for homonuclear molecules.

In Table 1, where we gathered useful data in the present context for diatomic systems of elements of the first four periods, except the rare gas and $Z n$; we also report the ratio $Q / Z$, equal to the relative contribution of the promolecular electronic force $F_{0}$ to the total force $Z^{2} / R_{e}{ }^{2}$.

Table 1. Atomic number $Z$, equilibrium distance $R_{e}$ (bohr), dissociation energy $D_{e}(\mathrm{kcal} / \mathrm{mol})$, deformation force $\Delta F(a u)$ and ratio $Q / Z$ for a series of diatomic molecules; $Q$ is the (calculated) total electronic charge contained in a sphere of radius $R_{e}$ in the isolated atom.

\begin{tabular}{|c|c|c|c|c|c|c|c|c|c|}
\hline & $\mathrm{H}_{2}$ & $\mathrm{Li}_{2}$ & $\mathrm{Be}_{2}$ & $B_{2}$ & $C_{2}$ & $\mathbf{N}_{2}$ & $\mathrm{O}_{2}$ & $F_{2}$ & \\
\hline Z & 1 & 3 & 4 & 5 & 6 & 7 & 8 & 9 & \\
\hline $\mathbf{R}_{\mathbf{e}}$ & 1.398 & 5.051 & 4.724 & 3.005 & 2.348 & 2.075 & 2.281 & 2.680 & \\
\hline$D_{e}$ & 103.3 & 26.3 & 15.0 & 67.0 & 145.0 & 226.0 & 118.9 & 38.0 & \\
\hline$Q / Z$ & 0.531 & 0.930 & 0.971 & 0.909 & 0.890 & 0.895 & 0.952 & 0.986 & \\
\hline$\Delta \mathbf{F}$ & 0.240 & 0.025 & 0.021 & 0.253 & 0.718 & 1.192 & 0.588 & 0.159 & \\
\hline & & $\mathrm{Na}_{2}$ & $\mathrm{Mg}_{2}$ & $\mathrm{Al}_{2}$ & $\mathrm{Si}_{2}$ & $\mathbf{P}_{2}$ & $S_{2}$ & $\mathrm{Cl}_{2}$ & \\
\hline Z & & 11 & 12 & 13 & 14 & 15 & 16 & 17 & \\
\hline $\mathbf{R}_{\mathbf{e}}$ & & 5.818 & 7.360 & 4.660 & 4.244 & 3.577 & 3.570 & 3.755 & \\
\hline$D_{e}$ & & 17.6 & 2.0 & 32.0 & 78.0 & 116.0 & 101.0 & 57.2 & \\
\hline$Q / Z$ & & 0.985 & 0.998 & 0.979 & 0.981 & 0.974 & 0.982 & 0.992 & \\
\hline$\Delta \mathbf{F}$ & & 0.054 & 0.004 & 0.167 & 0.208 & 0.460 & 0.367 & 0.169 & \\
\hline & & $K_{2}$ & $\mathrm{Ca}_{2}$ & $\mathrm{Ga}_{2}$ & $\mathrm{Ge}_{2}$ & $A s_{2}$ & $\mathrm{Se}_{2}$ & $\mathrm{Br}_{2}$ & \\
\hline $\mathbf{Z}$ & & 19 & 20 & 31 & 32 & 33 & 34 & 35 & \\
\hline $\mathbf{R}_{\mathbf{e}}$ & & 7.379 & 8.082 & 5.190 & 4.769 & 3.974 & 4.093 & 4.310 & \\
\hline$D_{e}$ & & 13.0 & 3.0 & 25.7 & 62.2 & 90.7 & 67.0 & 45.4 & \\
\hline$Q / Z$ & & 0.992 & 0.998 & 0.995 & 0.995 & 0.991 & 0.994 & 0.997 & \\
\hline$\Delta \mathbf{F}$ & & 0.052 & 0.015 & 0.179 & 0.243 & 0.646 & 0.436 & 0.216 & \\
\hline & $\mathrm{Sc}_{2}$ & $\mathrm{Ti}_{2}$ & $V_{2}$ & $\mathrm{Cr}_{2}$ & $\mathrm{Mn}_{2}$ & $\mathrm{Fe}_{2}$ & $\mathrm{CO}_{2}$ & $\mathrm{Ni}_{2}$ & $\mathrm{Cu}_{2}$ \\
\hline Z & 21 & 22 & 23 & 24 & 25 & 26 & 27 & 28 & 29 \\
\hline $\mathbf{R}_{\mathbf{e}}$ & 5.197 & 3.670 & 3.369 & 3.173 & 6.425 & 3.817 & 3.749 & 4.072 & 4.193 \\
\hline$D_{e}$ & 24.0 & 35.5 & 63.4 & 35.3 & 6.9 & 26.5 & 39.0 & 47.0 & 48.0 \\
\hline$Q / Z$ & 0.982 & 0.956 & 0.953 & 0.968 & 0.998 & 0.978 & 0.979 & 0.987 & 0.992 \\
\hline$\Delta \mathbf{F}$ & 0.297 & 1.585 & 2.172 & 1.817 & 0.037 & 1.035 & 1.066 & 0.632 & 0.409 \\
\hline
\end{tabular}


The major part of the total electronic attraction is provided by the promolecular density: $Q / Z$ is in most of case superior to $95 \%$ and never less than $89 \%$, except the case of $\mathrm{H}_{2}(53 \%)$. A general trend observed for main group elements is the increase of $Q / Z$ as the period increases: 0.53 for $\mathrm{H}_{2} \approx 0.90-0.99$ for $\mathrm{Li}_{2}-\mathrm{F}_{2}, 0.97-0.99$ for $\mathrm{Na}_{2}-\mathrm{Cl}_{2}$ and more than 0.99 for $\mathrm{K}_{2}-\mathrm{Br}_{2}$. Indeed, the number of core electrons which are near a total screen increases with respect to the total number $Z$ of electrons. In contrast to main group elements of the same period $\left(\mathrm{K}_{2}-\mathrm{Br}_{2}\right), \mathrm{Q} / \mathrm{Z}$ has a relatively wider range of variation $(\approx 0.95$ to 0.99$)$ in the transition element series. Smaller values are encountered for $\mathrm{Ti}_{2}$ and $\mathrm{V}_{2}$ which is consistent with both a more diffuse electron density and a shorter $R_{\mathrm{e}}$.



Fig. 1. Deformation forces $\Delta F(a u)$ and dissociation energy $D_{e}(\mathrm{kcal} / \mathrm{mol} / 100)$ as a function of the atomic number $Z$ in a series of homonuclear diatomic molecules.

Fig. 1 pictorially displays the variation of $\Delta \mathrm{F}$ with $\mathrm{Z}$ along with the corresponding dissociation energy. For main group elements, the same general pattern is observed within each period, namely $\mathrm{Li}_{2}-\mathrm{F}_{2}, \mathrm{Na}_{2}-\mathrm{Cl}_{2}$ and $\mathrm{K}_{2}-\mathrm{Br}_{2}$, with a rough parallelism of $\Delta \mathrm{F}$ and $\mathrm{D}_{\mathrm{e}}$ : stronger bonds correspond to greater $\Delta \mathrm{F}$. But in the $4^{\text {th }}$ period $\mathrm{K}_{2}-\mathrm{Br}_{2}, \Delta \mathrm{F}$ is systematically greater than for the corresponding elements of $\mathrm{Na}_{2}-\mathrm{Cl}_{2}$, though the $\mathrm{D}_{\mathrm{e}}$ are smaller. In the family of the transition elements, the situation is quite different. First, the parallelism of $\Delta F$ and $D_{e}$ is no longer respected in the sequence $\mathrm{Mn}_{2}-\mathrm{Cu}_{2}$; second, very large $\Delta \mathrm{F}$ are necessary to ensure relatively weak bonds, such as $\mathrm{Ti}_{2}, \mathrm{~V}_{2}$, or $\mathrm{Cr}_{2}$. 
Fig. 2 details the correlation between $\Delta \mathrm{F}$ and the bond dissociation energy De. A roughly linear correlation is observed for the main group elements as a whole. A very good correlation is obtained $\left(r^{2}=0.99\right)$ in the $\mathrm{Li}-\mathrm{F}$ row, as reported by Bader [12]. The correlation coefficient decreases as the period increases: 0.92 for $\mathrm{Na}-\mathrm{F}$ and 0.88 for $\mathrm{K}-\mathrm{Br}$. The correlation completely vanishes $\left(r^{2}=0.37\right)$ for the transition-metal series.

Indeed, there is no theoretical ground for an exact relationship between $\Delta F$ and $D_{e}$. At the most, we can infer that, since stronger bonds are generally shorter (counter examples may be found in Ref [27] and references therein), as the screening charge $Q$ is smaller, which needs an increase of $\Delta \mathrm{F}$. But even this qualitative assumption does not hold for the family of the transition metals: for example $\Delta \mathrm{F}=0.41$ for $\mathrm{Cu}_{2}\left(\mathrm{D}_{\mathrm{e}}=47.5 \mathrm{kcal} / \mathrm{mol}\right)$ and $\Delta \mathrm{F}=1.81$ for $\mathrm{Cr}_{2}$ $\left(D_{e}=35.3 \mathrm{kcal} / \mathrm{mol}\right)$.

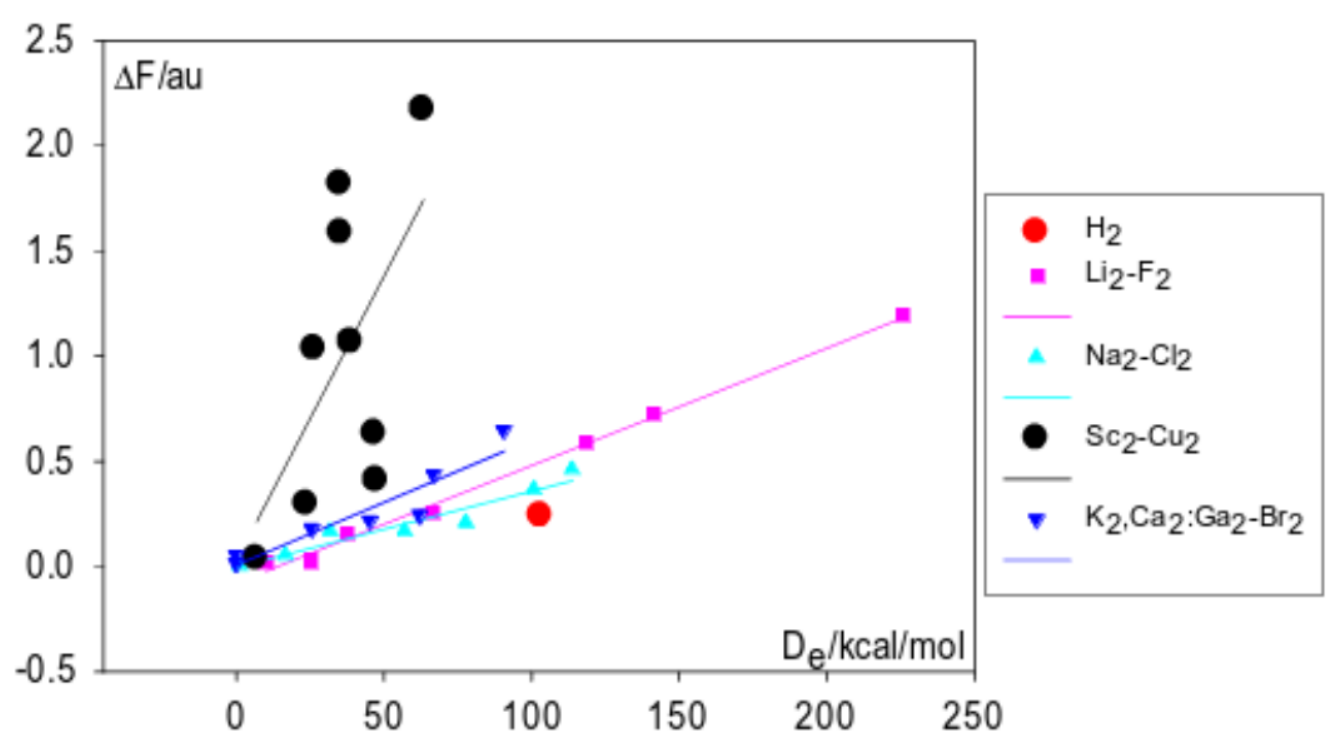

Fig. 2. Correlation of the deformation force $\Delta F$ with the dissociation energy of homonuclear diatomic molecules.

\subsection{Deformation force of the proionic density}

We considered as the proionic system the density of an ion pair, according to $A^{+} A^{-} \leftrightarrow A^{-} A^{+}$

Setting $Q^{+}$and $Q^{-}$the total electronic charge contained into a sphere of radius $R_{e}$ in the cation $\mathrm{A}^{+}$and the anion $\mathrm{A}^{-}$respectively, the proionic charge to be considered is 
$Q_{i}=1 / 2\left(Q++Q^{-}\right)$.

The proionic deformation force $\Delta \mathrm{F}^{\mathrm{i}}$ is thus:

$$
\Delta F^{i}=-\frac{Z}{R_{e}^{2}}\left[Z-\frac{1}{2}\left(Q^{+}+Q^{-}\right)\right] \quad \text { (eq. 2) }
$$

Note that because $Q^{+}$and $Q^{-}$are less than $Z-1$ and $Z+1$ respectively, the proionic charge is less than $\mathrm{Z}$, so that $\Delta \mathrm{F}^{\mathrm{i}}$ becomes negative, like the promolecular deformation force $\Delta \mathrm{F}$. We limited ourselves in these calculations to $\mathrm{H}_{2}$, the first row molecules the anion $\mathrm{A}^{-}$of which is stable: $B_{2}, C_{2}, N_{2}, O_{2}$, and all three halogen dimers $F_{2}, C l_{2}$ and $\mathrm{Br}_{2}$. The results are reported in Table 2.

Table 2. Ratio $Q^{i} / Z\left(Q^{i}\right.$ is the total average electronic charge of $A^{+}$and $A^{-}$contained is a sphere of radius $R_{e}$ ), promolecular $\Delta F$ and proionic $\Delta F^{i}$ deformation forces (au), and for a series of diatomic molecules.

\begin{tabular}{ccccccccc}
\hline & $\mathbf{H}^{+} \mathbf{H}^{-}$ & $\mathbf{B}^{+} \mathbf{B}^{-}$ & $\mathbf{C}^{+} \mathbf{C}^{-}$ & $\mathbf{N}^{+} \mathbf{N}^{-}$ & $\mathbf{O}^{+} \mathbf{O}^{-}$ & $\mathbf{F}^{+} \mathbf{F}^{-}$ & $\mathbf{C l}^{+} \mathbf{C l}^{-}$ & $\mathbf{B r}^{+} \mathbf{B r}^{-}$ \\
\hline $\mathbf{Q}^{\mathrm{i}} / \mathbf{Z}$ & 0.301 & 0.867 & 0.859 & 0.870 & 0.936 & 0.935 & 0.987 & 0.995 \\
$\Delta \mathbf{F}^{\mathrm{i}}$ & 0.358 & 0.370 & 0.919 & 1.483 & 0.788 & 0.731 & 0.265 & 0.329 \\
$\Delta \mathbf{F}$ & 0.240 & 0.253 & 0.718 & 1.192 & 0.588 & 0.159 & 0.169 & 0.216 \\
$\Delta \mathbf{F} / \Delta \mathbf{F}^{\mathbf{i}}$ & 0.671 & 0.685 & 0.781 & 0.804 & 0.746 & 0.218 & 0.636 & 0.655 \\
\hline
\end{tabular}

The most striking result is that $\Delta \mathrm{F}^{\mathrm{i}}$ is always greater than $\Delta \mathrm{F}$ which means that the proionic attractive force is less than the promolecular force, and thus that

$$
\mathrm{Q}^{+}+\mathrm{Q}^{-}<2 \mathrm{Q}
$$

The ratio $\Delta F / \Delta F^{i}$ is almost constant ca. $0.6-0.8$, except for $F_{2}(0.218)$ : the proionic density of $\mathrm{F}_{2}$ is less binding, with respect to the promolecular density, than in the other molecules of the series considered. 


\subsection{Vibrational frequencies of diatomic molecules}

Quantities in closer relation to the force than the dissociation energy are vibrational frequencies, as derivatives of the interatomic potential around the potential minimum. We may try thus to estimate the vibrational frequency of a diatomic molecule from the promolecular force: we saw that the total force $F_{R}$ is the sum of the promolecular force and the deformation force. Let us suppose for the moment that the deformation force is locally an additive constant to the repulsive promolecular force. For obtaining the derivative of the total force, we may thus calculate just the derivative of the promolecular force

$$
F_{\text {promolecular }}=\left(\frac{Z}{R}\right)^{2}\left(1-\frac{Q(R)}{Z}\right)
$$

from the atomic densities for homonuclear molecules with $Q$ being a function of the interatomic distance. Trying this for $\mathrm{N}_{2}$ we have second derivatives of the interatomic potential of 1.583 (B3LYP), 1.447 (open-shell multiconfigurational SCF, MCSCF) or 1.437 (adding correlation to MCSCF: MR-ACPF) around the potential minimum at $\mathrm{R}_{\mathrm{e}}=2.09 \mathrm{bohr}$, to be compared to the numerical derivative of the promolecular force at the experimental distance (2.075 bohr) from the integrated atomic Hartree-Fock densities of 1.666 (all in atomic units).

Leaving as additional simplification $Q$ a constant $Q\left(R_{\mathrm{e}}\right)$ around the equilibrium geometry, the derivative of the promolecular force with respect to $\mathrm{R}$ yields

$$
F_{\text {promolecular }}=2\left(\frac{Z^{2}}{R^{3}}\right)\left(1-\frac{Q\left(R_{e}\right)}{Z}\right)
$$

$=1.138$ for $\mathrm{Q}=6.27$ at $\mathrm{R}_{\mathrm{e}}=2.075$ bohr.

We may thus carry on the calculations through the whole second and third row of homonuclear dimers, and we find indeed a good correlation between experimental and calculated data, as depicted in Figure 3, following the observed trend of overestimated frequencies for the simple derivatives, and underestimated ones for the assumption of a constant promolecular charge. Interestingly, the procedure works even for the relatively weakly bound dimers $\mathrm{Be}_{2}$ and $\mathrm{Mg}_{2}$, as the main input is the experimental bond length, and 
as well for the highest frequency of molecular hydrogen. (Experimental values are from Irikura [28] but for $\mathrm{Li}_{2}$ [29] and $\mathrm{Al}_{2}$ [30]).

Table 2. Experimental and calculated vibration frequencies $\left(\mathrm{cm}^{-1}\right)$.

\begin{tabular}{llll}
\hline Molecule & Exp. & Q(R) & Q constant \\
\hline $\mathrm{H}_{2}$ & 4401 & 5540 & 4316 \\
$\mathrm{Li}_{2}$ & 351.4 & 452.1 & 272.4 \\
$\mathrm{Be}_{2}$ & 222.6 & 438.8 & 228.5 \\
$\mathrm{~B}_{2}$ & 1035.0 & 1514.4 & 873.3 \\
$\mathrm{C}_{2}$ & 1855.0 & 2750.6 & 1675.4 \\
$\mathbf{N}_{2}$ & 2358.6 & 2511.2 & 2076.0 \\
$\mathrm{O}_{2}$ & 1580.2 & 2349.0 & 1291.5 \\
$\mathrm{~F}_{2}$ & 916.9 & 1085.5 & 542.8 \\
$\mathrm{Na}_{2}$ & 159.1 & 359.2 & 206.1 \\
$\mathrm{Mg}_{2}$ & 51.12 & 112.2 & 51.6 \\
$\mathrm{Al}_{2}$ & 354 & 660.1 & 374.8 \\
$\mathrm{Si}_{2}$ & 511.0 & 808.1 & 442.6 \\
$\mathrm{P}_{2}$ & 780.8 & 1222.4 & 669.3 \\
$\mathrm{~S}_{2}$ & 725.7 & 1098.1 & 582.8 \\
$\mathrm{Cl}_{2}$ & 560 & 766.5 & 383.2 \\
\hline
\end{tabular}




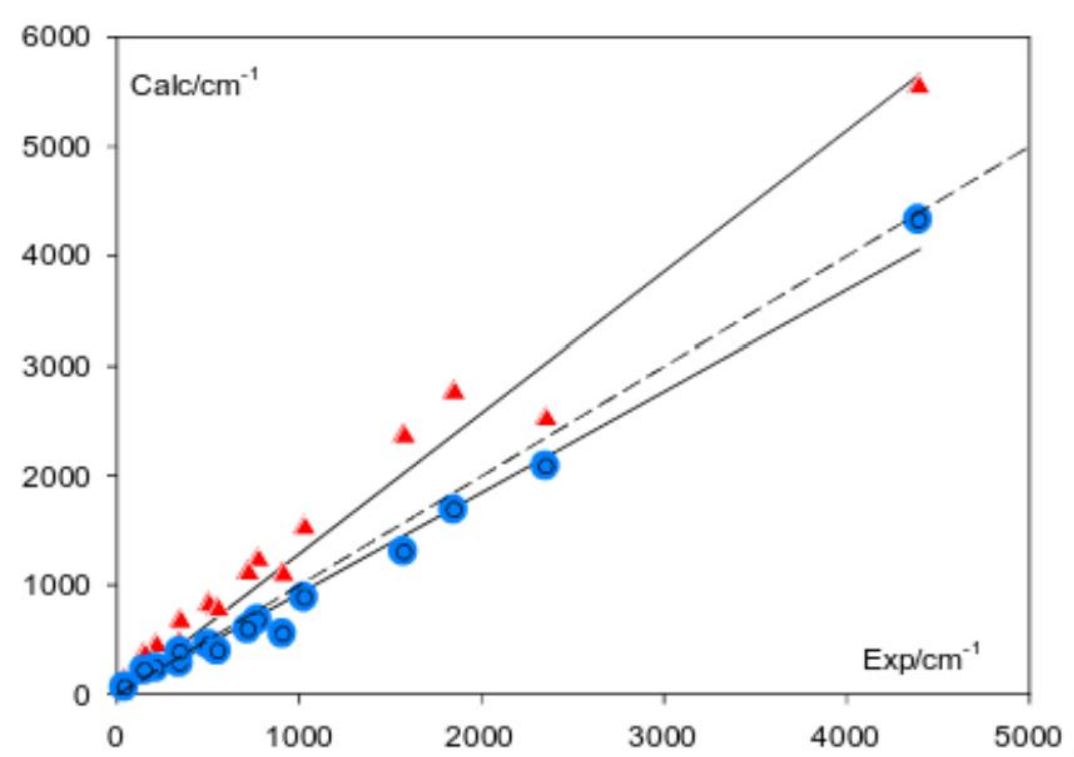

Figure 3. Calculated vibration frequencies vs. experimental values, for homonuclear diatomic molecules of Table 2 in $\mathrm{cm}^{-1}$. Red triangles refer to derivatives of $F$ taking into account the variation of $Q(R)$, blue circles stand for constant Qs.

Vibrational frequencies can be computed on the same basis for heteronuclear diatomic molecules. From Eq. 1, we obtain the deformation forces on the two atoms, for an internuclear distance $\mathrm{R}_{\mathrm{e}}$ :

$$
\Delta F=\frac{-Z_{A}}{R_{e}}\left(Z_{B}-Q_{B}\right)=\frac{-Z_{B}}{R_{e}}\left(Z_{A}-Q_{A}\right)
$$

The equality can be satisfied, similar to Eq. 2 , when replacing $Z_{A} Q_{B}$ and $Z_{B} Q_{A}$ by the average $1 / 2\left(Z_{A} Q_{B}+Z_{B} Q_{A}\right)$. Again we suppose that the deformation force varies only slightly with the interatomic distance, and all distance dependence of the total force is due to the promolecular (compensating) part only, where the charges $Q_{A}$ and $Q_{B}$ are as well assumed to be constant:

$F_{\text {promolecular }}=\frac{1}{R^{2}}\left[Z_{A} Z_{B}-\frac{1}{2}\left(Z_{B} Q_{A}+Z_{A} Q_{B}\right)\right]$

Thus the only term being differentiated is the $1 / R^{2}$ factor. This we try for an ensemble of halides, acids and hydrides. 




Fig. 4. Calculated vs. experimental vibrational frequencies of heteronuclear diatomic molecules. The lowest-frequency species are $\mathrm{KBr}, \mathrm{KCl}, \mathrm{NaBr}, \mathrm{NaCl}, \mathrm{LiBr}$ and $\mathrm{NaF}$.

Again, a good correlation is found with experimental data.

\section{Generalization for larger molecules}

For more than two atoms we may extend the promolecular force on atom $i$ to

$$
\vec{F}_{\text {promolecular }}=\sum_{j \neq i} \frac{Z_{i} Z_{j}}{R_{i j}^{2}}\left(\frac{\vec{R}_{i j}}{R_{i j}}\right)-\frac{1}{2} \sum_{j \neq i} \frac{Z_{i} Q_{j+} Z_{j} Q_{i}}{R_{i j}^{2}}\left(\frac{\vec{R}_{i j}}{R_{i j}}\right)=\sum_{j \neq i} \frac{K_{i j}}{R_{i j}^{2}}\left(\frac{\vec{R}_{i j}}{R_{i j}}\right)
$$

where we used the same symmetrization already employed before for heteronuclear diatomic molecules, introduced directions vectors, and collected the (effective) charges in a constant $K_{i j}$, assuming that the charges $Q_{\mathrm{j}}$ and $Q_{\mathrm{i}}$ remain nearly constant upon variation of the interatomic distance. The total force on atom $\mathrm{i}$ becomes thus

$$
\vec{F}_{i}=\sum_{i \neq j} K_{i j}\left(\frac{1}{R_{i j}^{2}}-\frac{1}{R_{e, i j}^{2}}\right)\left(\frac{\vec{R}_{i j}}{R_{i j}}\right)
$$

which we have to derive with respect to the atomic coordinates and evaluate at the equilibrium distance. From the product rule we see that again the only surviving derivative is that of the $1 / R^{2}$ terms. The symmetry of atomic coordinates in $\mathrm{R}$ leads to a real symmetric 
matrix when assembling the derivatives of the forces in mass-weighted coordinates to the Hessian matrix of the total energy. The frequencies are obtained as the eigenvalues, and modes as the eigenvectors.

The results for $\mathrm{H}_{2} \mathrm{O}, \mathrm{CO}_{2}, \mathrm{CS}_{2}$ and $\mathrm{HCN}$ are displayed in Table 3.

Table 3. Calculated and experimental vibration frequencies $\left(\mathrm{cm}^{-1}\right)$ for triatomic molecules

\begin{tabular}{llllllll}
\hline \multicolumn{2}{c}{$\mathbf{H}_{\mathbf{2}} \mathbf{O}$} & \multicolumn{2}{c}{$\mathrm{CO}_{\mathbf{2}}$} & \multicolumn{2}{c}{$\mathbf{C S}_{\mathbf{2}}$} & \multicolumn{2}{c}{$\mathrm{HCN}$} \\
\hline calc. & exp. & calc. & exp. & calc. & exp. & calc. & exp. \\
372.6 & 1595 & 0.000 & 665 & 0.000 & 397 & 0.000 & 712 \\
4180.5 & 3657 & 1226.2 & 1373 & 549.0 & 567 & 1903.0 & 2089 \\
4222.8 & 3756 & 2346.7 & 2349 & 1377.4 & 1535 & 3050.4 & 3312 \\
\hline
\end{tabular}

The most salient result is that if the stretching mode vibrations are reproduced within an error of ca. $10 \%$, the frequency of the deformation mode is strongly underestimated for $\mathrm{H}_{2} \mathrm{O}$ and is even vanishing for linear molecules. This result was expected: as a matter of fact, in a $\mathrm{AX}_{2}$ group, as long as atom $\mathrm{A}$ is modelled by a spherical density, the force constant for XAX angle variation arises only from the X...X promolecular force. This quantity does not vary when the interatomic distance is kept constant as for a bending mode in a linear setup, and becomes small for a molecule like $\mathrm{H}_{2} \mathrm{O}$ as the density decays exponentially with the interatomic distance. Thus, only vibrations which involve a significant variation of atom separation and thus of promolecular forces can be properly computed by this method. 

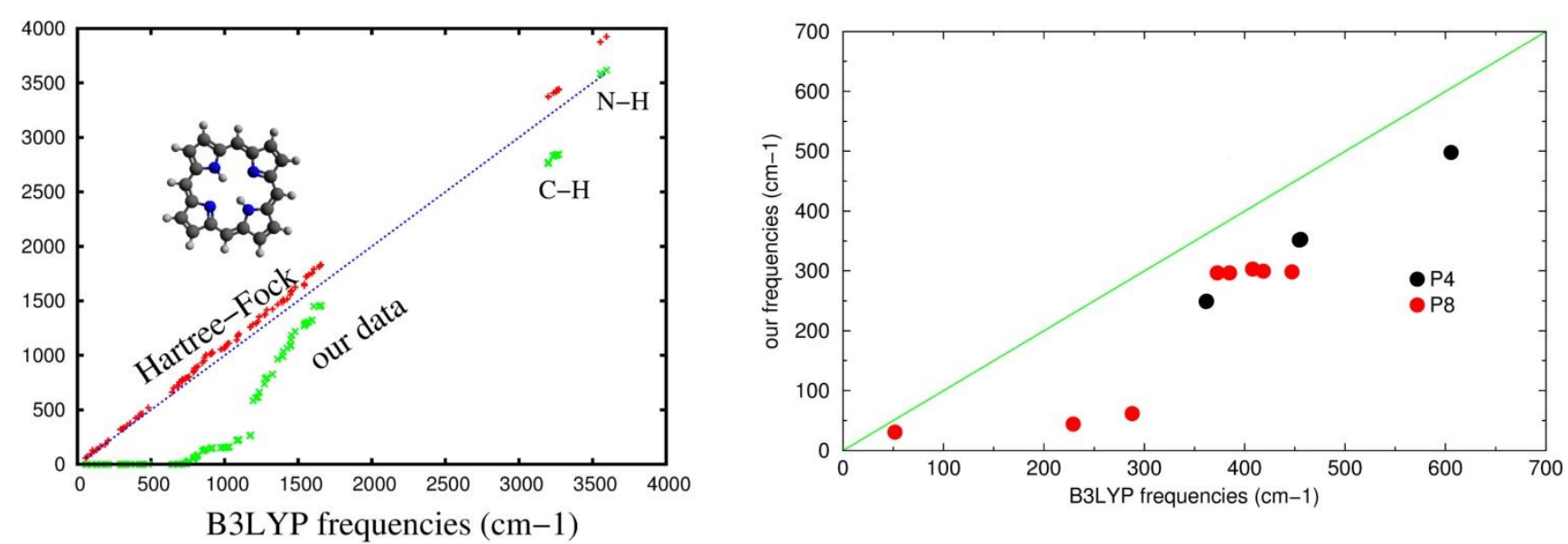

Fig. 5. Left panel: calculated data for porphyrine compared to DFT and HF results. Right panel: $P_{4}$ and $P_{8}$ frequencies with respect to B3LYP calculations.

The same features are observed in larger systems as different as porphyrine (Fig. 5), $\mathrm{P}_{4}$ and $P_{8}$ (Fig 5), adamantane, cubane and tetrahedrane (Fig 6).

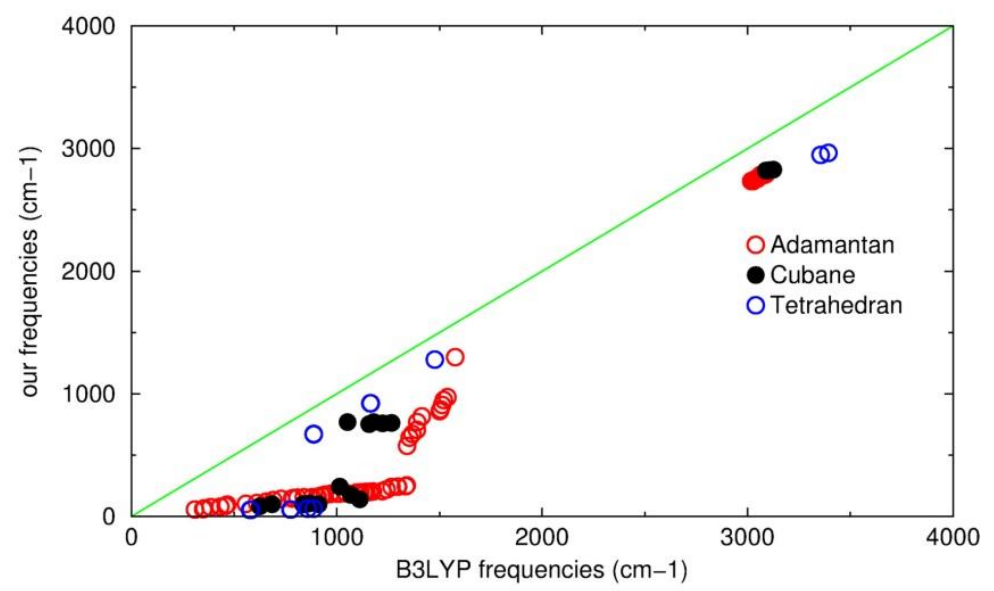

Fig. 6. Calculated data for three-dimensional hydrocarbons: adamantane, cubane and tetraedrane compared to DFT results.

For a given molecule, lower frequencies, which are associated to deformation modes are found much too small or nearly zero, while higher frequencies, associated to stretching modes are found with a precision close to Hartree-Fock calculations. Indeed, for the 3dimensional structures $\mathrm{P}_{4}$ where deformation modes are always associated which some bond-length variations, our approach gives satisfactory results. The same remark holds for carbon skeleton vibrations of tetrahedrane, cubane and adamantane. In these compounds, the series of near zero frequencies correspond to the $\mathrm{C}-\mathrm{H}$ bending modes. 
The method thus could be of interest for very large systems, allowing, with a very small computational effort and with the only data of promolecular densities and experimental bond lengths, the calculation of stretching (or dominantly stretching) frequencies modes of vibration. This may be interesting for still larger systems like proteins, where atomic positions and atom types are experimentally available, and for which our approach may yield reasonable dynamical features.

\section{Conclusion}

In this paper, we computed the force exerted by a spherically averaged atomic promolecular electron density on nuclei in a series of 30 diatomic molecules, ranging from $\mathrm{H}_{2}$ to $\mathrm{Br}_{2}$. Though 90 to $99 \%$ of the total force is provided by the promolecular density, the promolecule remains repulsive as expected.

We then report the deformation forces $\Delta \mathrm{F}$ associated to the deformation density $\Delta \rho$ in the molecule with respect to the promolecular density. For main group elements, $\Delta \mathrm{F}$ is roughly correlated to the dissociation energy, but the correlation decreases as the period increases. For transition metal dimers $\mathrm{Ti}_{2}-\mathrm{Cu}_{2}$, no correlation is obtained. Taking as reference a "proionic" density corresponding to a zwitterion, the proionic force is computed for a selected panel of 8 diatomic molecules. The proionic force found is less attractive than the promolecular one, especially in the case of $F_{2}$.

The combination of deformation forces and promolecular forces was used, via two essential approximations, to compute vibrational frequencies of diatomic molecules. The approach has been be generalized to polyatomic molecules, where it provides a rapid estimation of stretching (or dominantly stretching modes) vibrational frequencies with promolecular densities and experimental bond distances as only necessary input data.

\section{Acknowledgements}

It is a pleasure to recall discussions with A. Savin and B. Silvi (Paris) on the Silberbach controversy and the sphericity of atomic densities, and encouraging remarks of $\mathrm{YH}$ 
Sanejouand (CNRS Nantes). The laboratory of theoretical chemistry participates in the French excellence initiative via the project MiChem.

References:

[1] T.J. Berlin, Binding Regions in Diatomic Molecules, Chem. Phys., 19 (1951) 208

[2] The force concept in chemistry, B.M. Deb (Ed.), van Nostrand, New-York (1981)

[3] J. Fernández Rico, R. López, I. Ema, G. Ramírez, Chemical forces in terms of the electron density, Theor. Chem Acc. 118 (2007) 709

[4] Y. Ma, P. Politzer, Determination of noncovalent interaction energies from electronic densities, J. Chem. Phys. 120 (2004) 8955

[5] R. Balawender, F. De Proft, P. Geerlings, Nuclear Fukui function and Berlin's binding function: Prediction of the Jahn-Teller distortion, J. Chem. Phys. 114 (2001) 4441

[6] J. Fernández Rico, R. López, I. Ema, G. Ramírez, Chemical Notions from the Electron Density, Chem. Theory Comput. 1 (2005) 1083

[7] D. Chakraborty, C. Cárdenas, E. Echegaray, A. Toro-Labbe, P.W. Ayers, Understanding chemical binding using the Berlin function and the reaction force, Chem. Phys. Lett. 539 (2012) 168

[8] H. Silberbach, The electron density and chemical bonding: A reinvestigation of Berlin's theorem, J. Chem. Phys. 94 (1991) 2977

[9] J. Hinze, Comment on "The electron density and chemical bonding: A reinvestigation of Berlin's theorem", J. Chem. Phys. 101 (1994) 6369

[10] X. Wang, Z. Peng, Binding Regions in Polyatomic Molecules, Int. J. Quant. Chem. 47 (1993) 393

[11] S. Bratoz, R Daudel, M Roux, M Allavena, Some recent results concerning the electronic density and force constants of small molecules, Rev. Mod. Phys., 32 (1960) 412

[12] R.F.W. Bader, W.H. Henneken, Ionic Bond, J. Am. Chem. Soc. 87 (1965) 3063

[13] R.F.W. Bader, W.H. Henneker, P.E. Cade, Molecular Charge Distributions and Chemical Binding, J. Chem. Phys. 46 (1967) 3341

[14] R.F.W. Bader, A.D. Bandrauk, Molecular Charge Distribution and the Chemical Binding. 3. Isoelectronic series $\mathrm{N}_{2}, \mathrm{CO}, \mathrm{BF}$ and $\mathrm{C}_{2}, \mathrm{BeO}$, LiF", J. Chem. Phys. 49 (1968) 1653

[15] W.H.E. Schwarz, P. Valtazanos, K. Ruedenberg, Electron Difference Density and Chemical Bonding, Theor. Chim. Acta 68 (1985) 471 
[16] L. Mensching, W. von Niessen, P. Vattazanos, K. Ruedenberg, W.H.E. Schwarz, Chemical deformation densities. 2. Small molecules, J. Am. Chem. Soc. 111 (1989) 6933

[17] G. te Velde, F.M. Bickelhaupt, E.J. Baerends, C. Fonseca Guerra, S.J.A. van Gisbergen, J.G. Snijders, T. Ziegler, Chemistry with ADF, J. Comp. Chem. 22 (2001) 931

[18] F.L. Hirshfeld, S. Rzotkiewicz, Electrostatic binding in the first-row $\mathrm{AH}$ and $\mathrm{A}_{2}$ diatomic molecules, Mol. Phys. 27 (1974) 1319

[19] S. Shaik, P. Maître, G. Sini, P.C. Hiberty, The charge-shift bonding concept. Electron-pair bonds with very large ionic-covalent resonance energies, J. Am. Chem. Soc.114 (1992) 7861

[20] S. Shaik, D. Danovich, W. Wu, P.C. Hiberty, Charge-shift bonding and its manifestations in chemistry, Nature Chemistry 1 (2009) 443

[21] B. Roos, C. Salez, A. Veillard, E. Clementi, A general program for calculation of atomic SCF orbitals by the expansion method, IBM Tech Report RJ 518 (1968)

[22] F. Ruettea, M. Sánchez, R. Añeza, A. Bermúdez, A. Sierraalta, Diatomic molecule data for parametric methods. I, J. Mol Struc. THEOCHEM 729 (2005) 19

[23] S. Yanagisawa, T. Tsuneda, K. Hirao, An investigation of density functionals: The first-row transition metal dimer calculations, J. Chem. Phys. 112 (2000) 545

[24] J. Du, X. Sun, H. Wang, The confirmation of accurate combination of functional and basis set for transition-metal dimers: $\mathrm{Fe}_{2}, \mathrm{Co}_{2}, \mathrm{Ni}_{2}, \mathrm{Ru}_{2}, \mathrm{Rh}_{2}, \mathrm{Pd}_{2}, \mathrm{Os}_{2}, \mathrm{Ir}_{2}$, and $\mathrm{Pt}_{2}$, Int. J. Quant. Chem. 108 (2008) 1505

[25] J.E. Kingcade, H.M. Nagarathna-Naik, I. Shim, K.A. Gingerich, Electronic structure and bonding of the dimeric germanium molecule from all-electron ab initio calculations and equilibrium measurements, J. Phys. Chem. 90 (1986) 2830

[26] A. Scemama, IRPF90: a programming environment for high-performance computing, arXiv: 0909.5012v1

[27] E. Kraka, D. Setiawan, D. Cremer, Re-evaluation of the Bond Length-Bond Strength rule: the stronger bond is not always the shorter bond, J.Comp.Chem. 37 (2016) 130

[28] K.K. Irikura, Experimental Vibrational Zero-Point Energies: Diatomic Molecules, J. Phys. Chem. Ref. Data 36 (2007) 389

[29] T.H. Upton, Low-lying valence electronic states of the aluminum dimer, J. Phys. Chem. 90 (1986) 754

[30] B. Minaev, Ab initio study of low-lying triplet states of the lithium dimer, Spectrochimica Acta $162(2005) 790$ 